\title{
Space-charge-based electrostatic plasma confinement involving relaxed plasma species
}

\author{
J. L. Pacheco, C. A. Ordonez, a) and D. L. Weathers \\ Department of Physics, University of North Texas, Denton, Texas 76203, USA
}

(Received 8 August 2012; accepted 11 October 2012; published online 23 October 2012)

\begin{abstract}
A numerical study is reported on the equilibrium properties of a surface-emitted or edge-confined non-drifting plasma. A self-consistent finite-differences evaluation of the electrostatic potential is carried out for a non-neutral plasma that follows a Boltzmann density distribution. The non-neutral plasma generates an electrostatic potential that has an extremum at the geometric center. Poisson's equation is solved for different ratios of the non-neutral plasma size to the edge Debye length. The profiles of the electrostatic potential and the plasma density are presented for different values of that ratio. A second plasma species is then introduced for two-plasma-species confinement studies, with one species confined by the space charge of the other, while each species follows a Boltzmann density distribution. An equilibrium in which a neutral region forms is found. An equilibrium is also found in which the two species have equal temperatures and charge states. (C) 2012 American Institute of Physics. [http://dx.doi.org/10.1063/1.4764076]
\end{abstract}

\section{INTRODUCTION}

A variety of systems can provide electrostatic confinement of drifting non-neutral beams and plasmas, including the electrostatic storage ring, electrostatic ion beam trap, Kingdon trap, and electrostatic beam guide. ${ }^{1-18}$ However, electrostatic confinement of a non-drifting non-neutral singlespecies plasma is impossible, if such confinement requires using electrodes to produce a three-dimensional electrostatic potential well in vacuum. A three-dimensional electrostatic potential well can be produced by the space charge of a second species of particles of opposite charge sign. A variety of systems can provide space-charge-based electrostatic confinement of a non-drifting plasma species, including the electron beam ion trap/source, electron plasma ion trap/source, variable-electrode-radius Malmberg-Penning trap, nested Penning trap, inertial electrostatic confinement device, Penning fusion device, and a configuration consisting of a magnetic multipole superimposed on an electric multipole of higher order. $^{19-27}$

Suppose that a hollow and evacuated sphere, which is made from a refractory metal such as tungsten or tantalum, is heated to a temperature sufficient for thermionic electron emission to occur from the interior surface. A non-drifting non-neutral electron plasma would be produced within the interior of such a sphere. Under certain conditions, the space charge of that electron plasma can be used to confine a positive-ion plasma or a positron plasma. In the work presented here, the electrostatic potential and the density profile of a surface-emitted or edge-confined non-drifting nonneutral single-species plasma are self-consistently evaluated assuming a relaxed plasma. Next, the equilibrium of a two-species plasma, with one plasma species confined by the space charge of the other, is self-consistently evaluated.

\footnotetext{
a) Author to whom correspondence should be addressed. Electronic mail: cao@unt.edu.
}

Each species is assumed to be relaxed to a Boltzmann density distribution. An edge-confined plasma would be effectively unmagnetized, except near an outer boundary where a confining electromagnetic field would reside. ${ }^{28}$ One possibility is for the confining electromagnetic field to consist of a spatially periodic sequence of magnetic cusps that are plugged electrostatically. This is a case where a magnetic multipole would be superimposed on an electric multipole of higher order. The spatial period and range of the field would be much smaller than the dimensions of the plasma.

Figure 1 illustrates a type of configuration hereafter referred to as an artificially structured boundary. The field that would be produced by the configuration includes a sequence of electrostatically plugged coaxial magnetic ring cusps. The diameters of the ring cusps vary axially, with the smallest rings located near the axial ends of the confinement volume. Two point cusps that are coaxial with the ring cusps are located at the axial ends of the confinement volume. Notice that electrostatic plugging could be applied along straight magnetic field lines that connect with the magnetic cusps. The straight magnetic field lines that connect to each of the two point cusps would be associated with a uniform magnetic field, which could extend to regions where two Penning traps (not shown) could provide storage and cooling of non-neutral plasmas. Preliminary classical trajectory Monte Carlo studies suggest that a portion of the charged particles located near the axis of symmetry of the Penning traps could pass adiabatically from the magnetized region into the effectively unmagnetized region, thereby providing a means of loading and sustaining low-temperature plasmas within the effectively unmagnetized region.

A motivation for the work presented here is the prospect of testing fundamental symmetries between the properties of matter and antimatter such as the gravitational acceleration symmetry. ${ }^{29,30}$ Antihydrogen is currently produced and trapped by using nested Penning traps to 


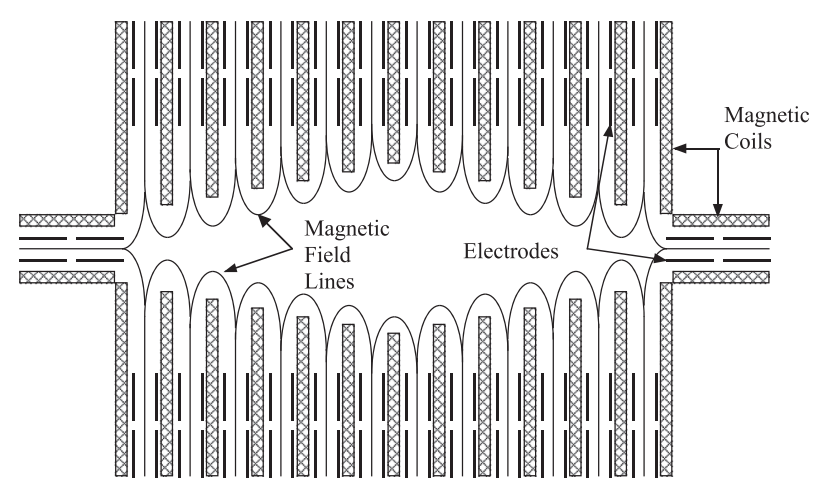

FIG. 1. Illustration of an axisymmetric artificially structured boundary that could serve to confine a non-neutral plasma species, which would provide space-charge-based electrostatic confinement of a second plasma species.

confine antihydrogen plasma. ${ }^{31,32}$ However, plasma drifts within nested Penning traps represent a formidable problem for producing antihydrogen with sufficiently low kinetic energy to be trapped in useful quantities for experimentation. ${ }^{33-35}$ An antihydrogen atom is born with the kinetic energy of its antiproton, and plasma drifts can increase the kinetic energy of antiprotons. An ideal plasma confinement approach for antihydrogen studies would avoid plasma drifts and be capable of providing long confinement times for a cold, dense, non-drifting (e.g., non-rotating) plasma of any desired size.

An artificially structured boundary could confine a nonneutral positron plasma, which in turn provides spacecharge-based electrostatic confinement of an antiproton plasma. For the concept illustrated in Fig. 1, the antiproton plasma would have a distorted (elongated) spheroidal shape. However, other geometries for space-charge-based electrostatic confinement could also be envisioned, including spherical and toroidal geometries. For studies that require trapped antihydrogen, a finite minimum B configuration could be produced by locating a current-carrying wire along the axis of symmetry, although it would complicate loading plasmas. For antihydrogen gravity studies, it is not necessary to trap antihydrogen atoms within a minimum-B magnetic field. If antihydrogen is produced within a configuration such as that illustrated in Fig. 1, antihydrogen atoms that leave radially or axially between electrodes could serve for such studies. Two things should be mentioned. The three-body recombination rate within a hydrogen (or antihydrogen) plasma can be about a factor of 10 larger within an unmagnetized plasma relative to a magnetized plasma, all other parameters being equal. ${ }^{36}$ Positive ions that can be laser cooled could be confined along with the positrons, or negative ions that can be laser cooled could be electrostatically confined with the antiprotons.

In Sec. II, a self-consistent computation of the electrostatic potential that occurs within an unmagnetized nonneutral plasma under equilibrium conditions is developed. In Sec. III, a second plasma species is introduced and the resulting equilibrium of a two-species plasma is evaluated. In Sec. IV, the conditions necessary for achieving space-chargebased electrostatic confinement are discussed. Concluding remarks are found in Sec. V.

\section{SINGLE-SPECIES NON-NEUTRAL PLASMA}

A region of space that contains electric field sources must satisfy Poisson's equation. Therefore, the electrostatic potential resulting from a Boltzmann distribution of charged particles can be obtained by solving Poisson's equation and imposing the appropriate boundary conditions according to the geometry of the problem. Poisson's equation (in SI units) reads

$$
\nabla^{2} \phi(\boldsymbol{r})=-\frac{\rho(\boldsymbol{r})}{\epsilon_{0}}
$$

where $\phi(\boldsymbol{r})$ is the electrostatic potential, $\rho(\boldsymbol{r})$ is the charge density, and $\epsilon_{0}$ the vacuum permittivity. Assume that a single-species plasma is in a steady-state equilibrium. Furthermore, assume that the plasma is relaxed, such that the Boltzmann density distribution represents the charged particle distribution in space. ${ }^{22}$ Also, let the electrostatic potential be represented by the average of the local electrostatic potential (averaging over the discreteness of the plasma constituents). The Boltzmann relation for the plasma density is

$$
n(\boldsymbol{r})=n_{s} e^{-q\left[\phi(\boldsymbol{r})-\phi\left(\boldsymbol{r}_{s}\right)\right] / T} .
$$

Here, $n_{s}$ is a known plasma density at $\boldsymbol{r}_{s}, q$ is the charge of a plasma particle (e.g., $q=-e$ for an electron plasma where $e$ is the unit charge), and $T$ is the plasma temperature in energy units. The plasma temperature is assumed to be temporally constant and spatially uniform. Equation (1) becomes

$$
\nabla^{2} \phi(\boldsymbol{r})=-\frac{q n_{s}}{\epsilon_{o}} e^{-q\left[\phi(\boldsymbol{r})-\phi\left(\boldsymbol{r}_{s}\right)\right] / T} .
$$

At the location where the plasma density is specified (i.e., at $\boldsymbol{r}_{s}$ ), the electrostatic potential is defined to be zero: $\phi\left(\boldsymbol{r}_{s}\right)=0$. By solving Eq. (3) for the electrostatic potential, the plasma equilibrium can be obtained. In order to generalize the study, the equation is normalized by introducing a dimensionless potential $\psi(\boldsymbol{r})=q \phi(\boldsymbol{r}) / T$, and defining the Debye length at $\boldsymbol{r}_{s}$ as $\lambda_{D}=\sqrt{\epsilon_{0} T /\left(q^{2} n_{s}\right)}$. With these modifications, the governing equation simplifies to

$$
\nabla^{2} \psi(\boldsymbol{r})=-\frac{e^{-\psi(\boldsymbol{r})}}{\lambda_{D}^{2}} .
$$

Equation (4) is solved for spherical, cylindrical, and planar geometries.

(1) For the spherical geometry, a system that has spherical symmetry is assumed. Let $r$ denote the radial coordinate of a spherical coordinate system. The governing equation reads

$$
\frac{2}{r} \frac{\partial \psi}{\partial r}+\frac{\partial^{2} \psi}{\partial r^{2}}=-\frac{e^{-\psi}}{\lambda_{D}^{2}} .
$$

(2) For the cylindrical geometry, a system is assumed that has infinite length in the axial dimension and is cylindrically symmetric. Let $r$ denote the radial coordinate of the cylindrical system. The governing equation in cylindrical coordinates is 


$$
\frac{1}{r} \frac{\partial \psi}{\partial r}+\frac{\partial^{2} \psi}{\partial r^{2}}=-\frac{e^{-\psi}}{\lambda_{D}^{2}}
$$

(3) For the planar geometry, assume that the system is contained between two infinite planes. Let the variable $r$ be defined as a Cartesian coordinate normal to the planes. A system that has mirror symmetry about $r=0$ is assumed. In this case, the governing equation is

$$
\frac{\partial^{2} \psi}{\partial r^{2}}=-\frac{e^{-\psi}}{\lambda_{D}^{2}} .
$$

In the previous three equations, $\psi$ is a function of the variable $r, \psi=\psi(r)$. The notation has been suppressed for brevity.

Equations (5)-(7) are combined into a single equation. By introducing a coefficient of the form $(\alpha-1) / r$ in place of the term multiplying the first partial derivative and changing variables to the spatial coordinate $r_{n}=r / \lambda_{D}$, the following equation is obtained:

$$
\frac{(\alpha-1)}{r_{n}} \frac{\partial \psi\left(r_{n}\right)}{\partial r_{n}}+\frac{\partial^{2} \psi\left(r_{n}\right)}{\partial r_{n}^{2}}=-e^{-\psi\left(r_{n}\right)}
$$

Here, $\alpha$ takes the value of 1,2 , or 3 for the planar, cylindrical, or spherical geometry, respectively. Thus, Eqs. (5)-(7) are simultaneously represented by Eq. (8) in terms of the normalized coordinate $r_{n}$.

\section{A. Boundary conditions}

The symmetry of the charge distribution dictates a set of mixed boundary conditions.

(1) Neumann boundary condition

The electric field is zero at the origin

$$
\left[\frac{\partial \phi(r)}{\partial r}\right]_{r=0}=\left[\frac{\partial \psi\left(r_{n}\right)}{\partial r_{n}}\right]_{r_{n}=0}=0 .
$$

(2) Dirichlet boundary condition

The electrostatic potential is defined to be zero at the plasma edge

$$
\phi\left(r_{\max }\right)=\psi\left(r_{n, \max }\right)=0 .
$$

Here, the plasma edge is located at $r_{\max }$ and at $r_{n, \max }=r_{\max } / \lambda_{D}$, where $\lambda_{D}$ is the Debye length at the plasma edge. The plasma diameter, or thickness in the planar geometry, is $2 r_{\max }$. The method by which the plasma is produced, sustained, and confined is not considered here. The description is only applicable for the region $0 \leq r \leq r_{\max }$.

\section{B. Finite differences}

A finite-differences computational approach has been used to predict plasma equilibria in nested-well and singlewell Malmberg-Penning traps. ${ }^{33,37}$ Equation (8) is solved using a finite-differences approach. Written in terms of finite differences, the first partial derivative of a general function, $f(x, y, z)$, with respect to $x$ (in symmetrical form) becomes

$$
\frac{\partial f(x, y, z)}{\partial x} \approx \frac{f(x+\Delta x, y, z)-f(x-\Delta x, y, z)}{2 \Delta x} .
$$

The second partial derivative is

$$
\frac{\partial^{2} f(x, y, z)}{\partial x^{2}} \approx \frac{f(x+\Delta x, y, z)+f(x-\Delta x, y, z)}{(\Delta x)^{2}}-\frac{2 f(x, y, z)}{(\Delta x)^{2}} .
$$

In principle, this recipe can be used to represent any second order partial differential equation. Applying the finitedifferences approach to Eq. (8) gives, after some algebraic manipulations,

$$
\begin{aligned}
\psi\left(r_{n}\right)= & \frac{\omega \Delta r_{n}^{2}}{2}\left[\frac{(\alpha-1)}{r_{n}} \frac{\psi\left(r_{n}+\Delta r_{n}\right)-\psi\left(r_{n}-\Delta r_{n}\right)}{2 \Delta r_{n}}\right. \\
& +\frac{\psi\left(r_{n}+\Delta r_{n}\right)+\psi\left(r_{n}-\Delta r_{n}\right)}{\Delta r_{n}^{2}} \\
& \left.+e^{-\psi\left(r_{n}\right)}\right]-(\omega-1) \psi\left(r_{n}\right),
\end{aligned}
$$

where the left-hand side represents the new value each iteration, and the normalized grid spacing is $\Delta r_{n}=\Delta r / \lambda_{D} . \omega$ is introduced for the purpose of reducing computation time. ${ }^{38}$ Equation (13) is implemented using a sequential overrelaxation method, with $\omega$ having a value in the range $1 \leq \omega<2$. $^{37}$

\section{Self-consistent solution}

A computer program was developed to solve for the normalized electrostatic potential $\psi$ using the finite-differences approach. The parameter $\omega$ and the number of iterations were chosen to achieve the desired convergence. The selfconsistent computation of the electrostatic potential was achieved by iteratively solving for the electrostatic potential according to Eq. (13). All computations were run until the absolute difference between one iteration and the next was less than $10^{-10}$ at every grid point. It has been reported that if the grid spacing is on the order of, or smaller than, the Debye length, code instabilities are reduced, and the convergence of a solution is more likely to be achieved. ${ }^{39}$ The computation assigned at least three grid points per Debye length for $r_{\max } \gg \lambda_{D}$ and significantly more grid points $(\approx 50)$ per Debye length for values of $r_{\max } \approx \lambda_{D}$.

Figure 2 shows typical profiles of the electrostatic potential. The three plots correspond to the planar, cylindrical, and spherical geometries ( $\alpha=1,2$, and 3, respectively). The non-neutral plasma generates an electrostatic potential that has an extremum at the center of each geometry (at $r_{n}=0$ ).

In Fig. 3, radial profiles of the normalized density function, $e^{-\psi}$, are shown. The profiles are for a spherical geometry, $\alpha=3$, with $r_{n, \max }=5,10,20$, and 30 . The non-neutral plasma density has a minimum at the geometric center of the system. The behavior of the plasma distribution in the vicinity of the boundary is observed to change in a more pronounced manner as the value of $r_{n, \max }$ increases, behavior 


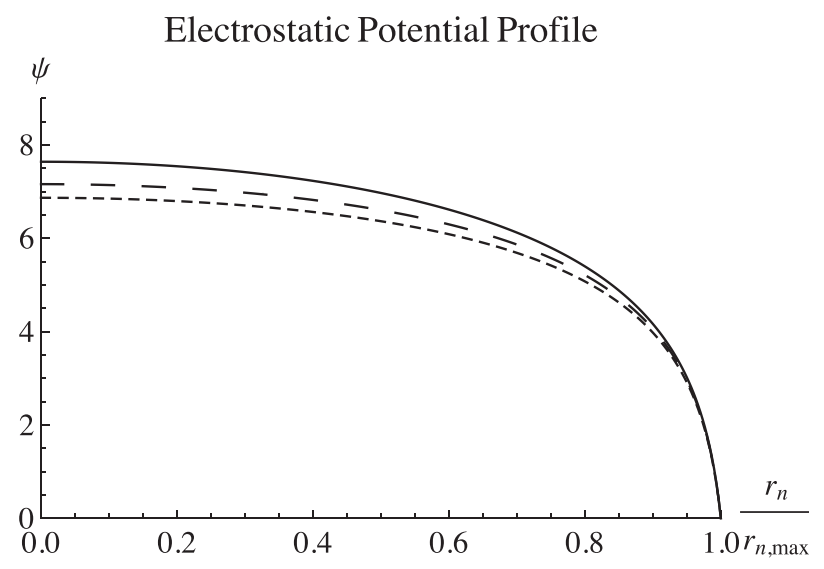

FIG. 2. Typical radial profiles of the normalized electrostatic potential. The plots are for $r_{n, \max }=100$ and $\alpha=1$ (solid), 2 (long dash), and 3 (short dash).

that agrees qualitatively with previous results for magnetized non-neutral plasmas. ${ }^{40}$

The behavior of the plasma has also been characterized by evaluating the normalized potential at the geometric center of the volume in question. Recalling the boundary condition $\psi\left(r_{n, \max }\right)=0$, the normalized potential at $r_{n}=0$ is equal to the difference in normalized potential between the geometric center and the boundary: $\Delta \psi_{0, \alpha}=\psi(0)-\psi$ $\left(r_{n, \max }\right)$. The value of the $\alpha$ subscript indicates the geometry being studied. Figure 4 shows how the normalized potential difference, $\Delta \psi_{0, \alpha}$, changes for increasing values of $r_{n, \max }$ for planar, cylindrical, and spherical geometries $(\alpha=1,2,3)$. The value increases rapidly for small values of $r_{n, \max }$ and increases at a much slower rate for larger values of $r_{n, \max }$. Notice that values exceeding 10 are predicted. Such large values indicate that it should be possible to confine a second species of particles with opposite sign of charge within the electrostatic potential well created by the first species, with both species having the same temperature and charge state. Such a possibility is considered in Sec. III.

The numerical results in Fig. 4 were fitted by an analytical expression. The approximate analytical expression

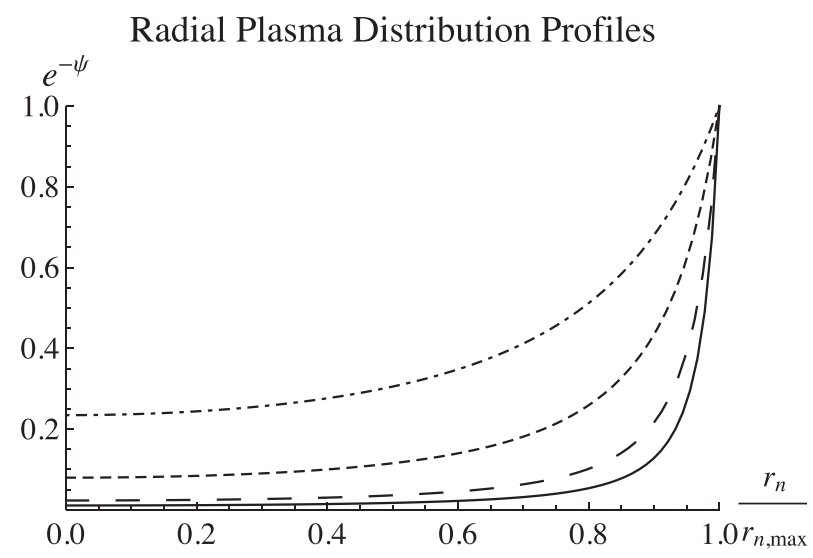

FIG. 3. Typical normalized density profiles. The plots are for $\alpha=3$ and $r_{n, \max }=5$ (dot-dashed), 10 (short dash), 20 (long dash), and 30 (solid). Similar profiles occur for other values of $\alpha$.

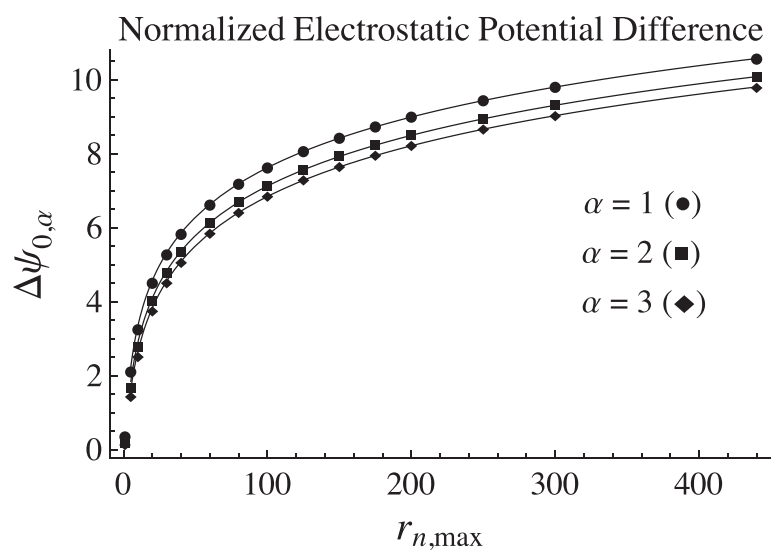

FIG. 4. Normalized electrostatic potential difference (between plasma center and edge) for the three geometries. The solid lines are Eq. (14).

obtained for the normalized electrostatic potential difference between the origin at $r_{n}=0$ and the boundary at $r_{n, \max }$ is

$$
\Delta \psi_{0, \alpha}=\ln \left[\left(r_{n, \max }\right)^{0.217 \alpha^{0.139}}\right]+W\left(\frac{r_{n, \max }^{2}}{2 \alpha}\right) .
$$

Here, $W(x)$ is the product logarithm function, which satisfies the equation $x=W(x) \exp [W(x)]$. The last term on the right of Eq. (14) is the functional dependence that is expected in the asymptotic limit of large $r_{n, \max }$. In such a limit, the density is spatially constant, except near the plasma edge, where the variation is ignored. Equation (14) agrees with numerical results to within $1 \%$ for $30 \leq r_{n, \max } \leq 440$ and to within $15 \%$ for $1 \leq r_{n, \max } \leq 30$. As a specific case, consider the electrostatic potential generated for $r_{n, \max }=100$ and $\alpha=3$. In such a case, $\Delta \psi_{0, \alpha}$ has a value of 6.85 , and a $1 \mathrm{eV}$ (temperature) electron plasma generates an electric potential difference between the plasma edge and center of $6.85 \mathrm{~V}$.

\section{TWO-SPECIES PLASMA}

Consider a surface-emitted or edge-confined electron plasma that follows a Boltzmann distribution as described in Sec. II. An electrostatic potential energy well is created for positive particles. Assume that a positive plasma species, such as a positive-ion or positron plasma, is introduced near the center of the electron plasma. The charge density is $\rho(r)$ $=-e n_{-}(r)+Z e n_{+}(r) . Z$ represents the average charge state of the positive plasma particles. The first term on the righthand side refers to the electron plasma, which follows a Boltzmann density profile of the form $n_{-}(r)=n_{0_{-}} \exp (e[\phi(r)$ $\left.\left.-\phi\left(r_{s}\right)\right] / T_{-}\right)$. The second term refers to the positive plasma that is confined by the electrostatic potential well created by the space charge of the electron plasma. The positive plasma is also assumed to follow a Boltzmann distribution: $n_{+}(r)$ $=n_{0_{+}} \exp \left(-Z e[\phi(r)-\phi(0)] / T_{+}\right)$. Poisson's equation is

$$
\nabla^{2} \phi(r)=-\frac{1}{\epsilon_{0}}\left[-e n_{0_{-}} e^{\frac{e\left[\phi(r)-\phi\left(r_{s}\right)\right]}{T_{-}}}+Z_{e} n_{0_{+}} e^{\frac{-Z e[\phi(r)-\phi(0)]}{T_{+}}}\right]
$$

where $n_{-}\left(r_{s}\right)=n_{0_{-}}$and $n_{+}(0)=n_{0_{+}}$are known densities of the respective plasma species. Let $N_{n}=Z n_{0_{+}} / n_{0_{-}}$and 
$T_{n}=Z T_{-} / T_{+} \cdot N_{n}$ is the positive plasma charge density at the center of the configuration normalized by the magnitude of the electron charge density at the edge. $T_{n}$ is the average charge state of the positive plasma particles multiplied by the ratio of the electron temperature to the temperature of the positive species. Write

$$
\nabla^{2} \phi(r)=-\frac{e n_{0_{-}}}{\epsilon_{0}}\left[-e^{\frac{e\left[\phi(r)-\phi\left(r_{s}\right)\right]}{T_{-}}}+N_{n} e^{\frac{-e T_{n}[\phi(r)-\phi(0)]}{T_{-}}}\right] .
$$

Introduce the normalized electrostatic potential, $\psi(r)=$ $e \phi(r) / T_{-}$, with $\phi\left(r_{s}\right)=0$, where $r_{s}$ defines the boundary of the system

$$
\nabla^{2} \psi(r)=-\frac{e^{2} n_{0_{-}}}{\epsilon_{0} T_{-}}\left[-e^{\psi(r)}+N_{n} e^{-T_{n}[\psi(r)-\psi(0)]}\right] .
$$

Define the Debye length at the boundary of the electron plasma as $\lambda_{D_{-}}^{2}=\left(\epsilon_{0} T_{-}\right) /\left(e^{2} n_{0_{-}}\right)$and scale the coordinates with respect to this quantity. Now,

$$
\nabla^{2} \psi\left(r_{n}\right)=e^{\psi\left(r_{n}\right)}-N_{n} e^{-T_{n}\left[\psi\left(r_{n}\right)-\psi(0)\right]},
$$

where $r_{n}=r / \lambda_{D_{-}}$is the normalized radial coordinate and $\psi(0)$ is the value of the normalized electrostatic potential at

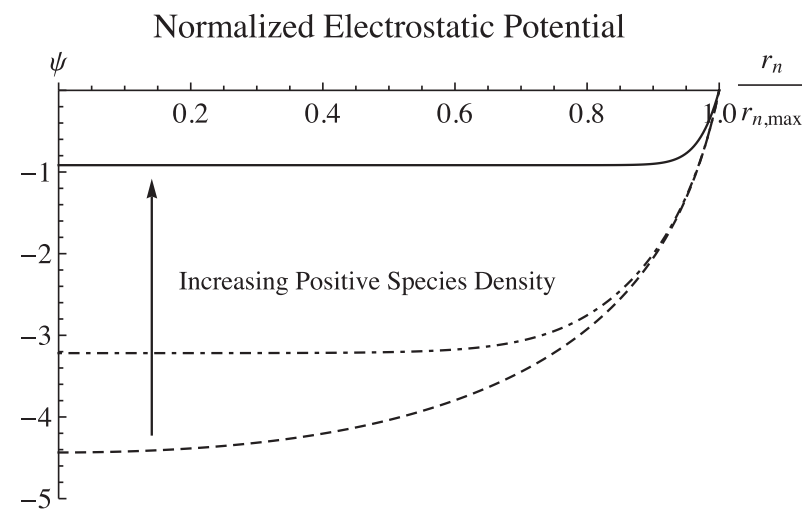

Two-Species Plasma Distribution

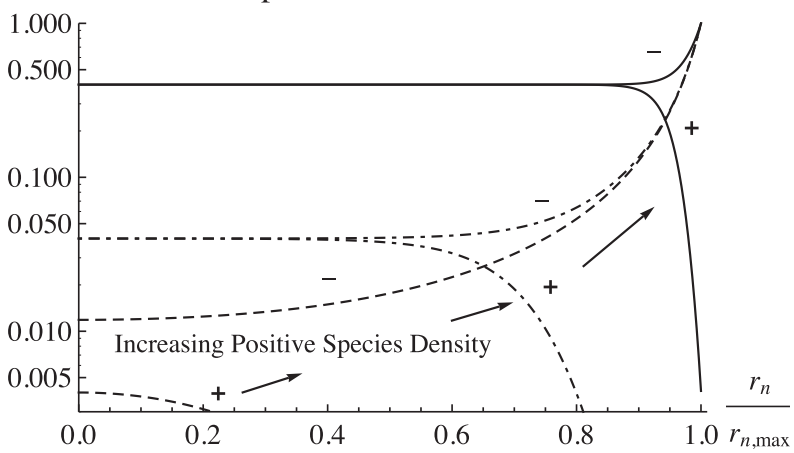

FIG. 5. Normalized electrostatic potential of a two-species plasma (top). Self-consistent distributions of the two plasma species (bottom) in logarithmic scale. The plots are for $T_{n}=5, r_{n, \max }=30$, and $N_{n}=0.004$ (dashed), 0.04 (dot-dashed), 0.4 (solid). The arrows indicate the trend that the system follows as $N_{n}$ is increased. In the lower panel of this figure and Figs. 6-8, the normalized distributions are $n_{-}\left(r_{n}\right) / n_{0_{-}}$, which are labeled by minus signs (-), and $Z n_{+}\left(r_{n}\right) / n_{0}$, which are labeled by plus signs $(+)$. Thus, each matching pair of plots are the normalized distributions for the negative and positive plasma species. the center of the system. The Laplacian can be replaced by the left-hand side of Eq. (8). The boundary conditions for the two-species plasma are identical to those for the singlespecies plasma computations presented in Sec. II, namely, Eqs. (9) and (10).

A finite-differences approach was used to selfconsistently evaluate the properties of the plasma system. The shape of the electrostatic potential profile must now adjust to include the effect of the positive species occupying the central region. Comparing Eq. (18) to Eq. (8), one can see that Eq. (13) is applicable here, except that the density term becomes

$$
e^{-\psi\left(r_{n}\right)} \rightarrow N_{n} e^{-T_{n}\left[\psi\left(r_{n}\right)-\psi(0)\right]}-e^{\psi\left(r_{n}\right)} .
$$

The computer program developed to solve for the normalized electrostatic potential for a two-species plasma configuration is similar to the one used for Sec. II, except that it was adapted to include the density term for the second plasma species. The number of grid points was determined by the smallest Debye length (smallest of the two species). At least three grid points per such Debye length were used. The normalized electrostatic potential was iteratively computed in a self-consistent manner until the absolute difference between one iteration and the next was less than $10^{-10}$ at every grid point. Initial results indicate that the electrostatic potential is similar for different geometries even when the positive plasma is introduced. Only computations

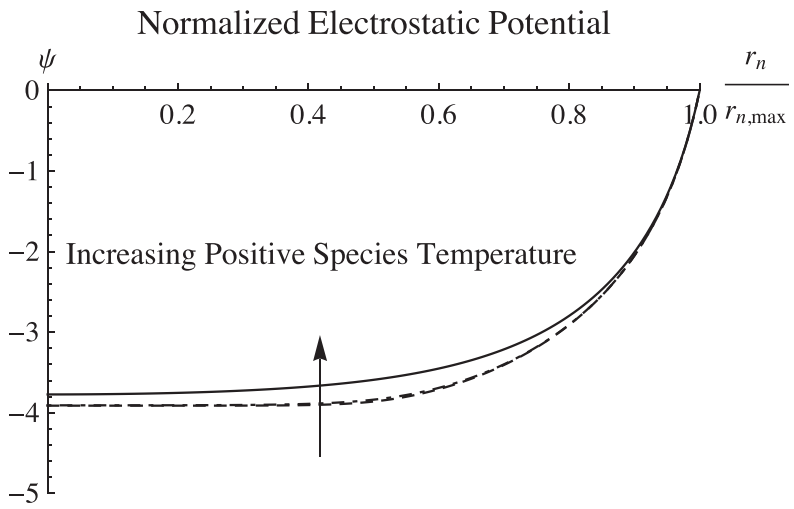

Two-Species Plasma Distribution

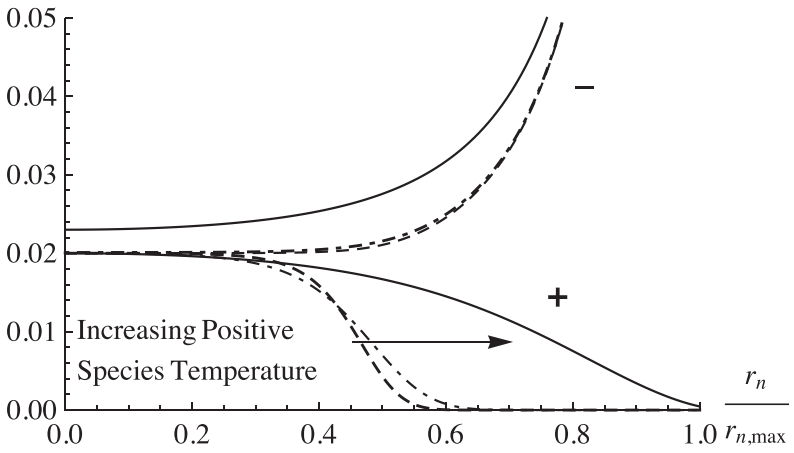

FIG. 6. Normalized electrostatic potential of a two-species plasma (top). Self-consistent distributions of the two plasma species (bottom). The plots are for $N_{n}=0.02, r_{n, \max }=30$, and $T_{n}=1$ (solid), 15 (dot-dashed), and 30 (dashed). The \pm labels are defined in Fig. 5. 
pertaining to a spherically symmetric system are presented, with the expectation that the results obtained are qualitatively applicable to the planar and cylindrical geometries.

The depth of the electrostatic potential well tends to decrease as the positive plasma charge density is increased. Such behavior is shown in Fig. 5. Also, for sufficiently high charge densities of the positive plasma, both species approach the same charge density at the center of the system. If the positive plasma charge density is sufficiently small, the electrostatic potential and density of the negative plasma are relatively unaffected by the presence of the positive plasma. However, Fig. 6 shows that, for such a case, the volume occupied by the positive plasma increases as the temperature of the positive plasma is increased, or its average charge state is decreased.

The normalized electrostatic potential and distribution profiles for two plasma species with equal temperatures and charge states under equilibrium conditions are shown in Fig. 7. Notice that for sufficiently high charge densities of the positive plasma, the system reaches neutrality near the center of the system. When the charge density of the positive plasma is sufficiently low, there exists a state of partial neutralization. Figure 8 shows the minimum positive species charge density at which the system achieves approximate neutrality at the center of the system for different temperatures or charge states of the two plasma species. The minimum neutral density decreases as the positive plasma temperature is lowered or its average charge state is increased.

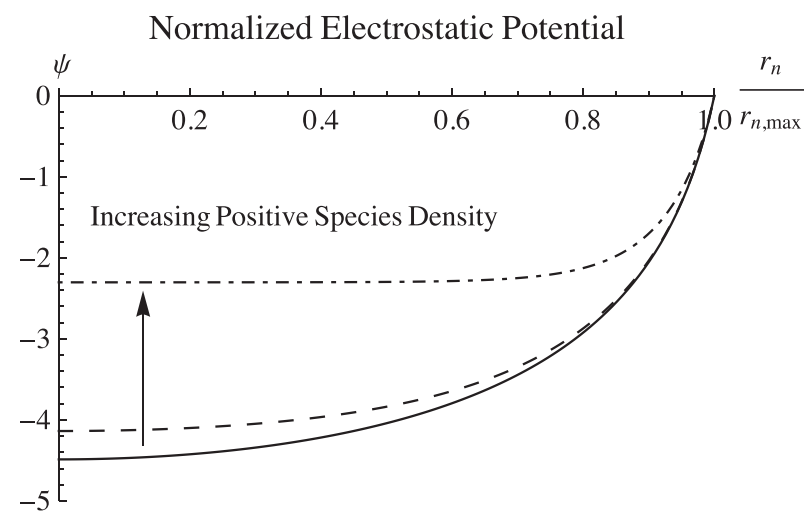

Two-Species Plasma Distribution: Equal Temperatures

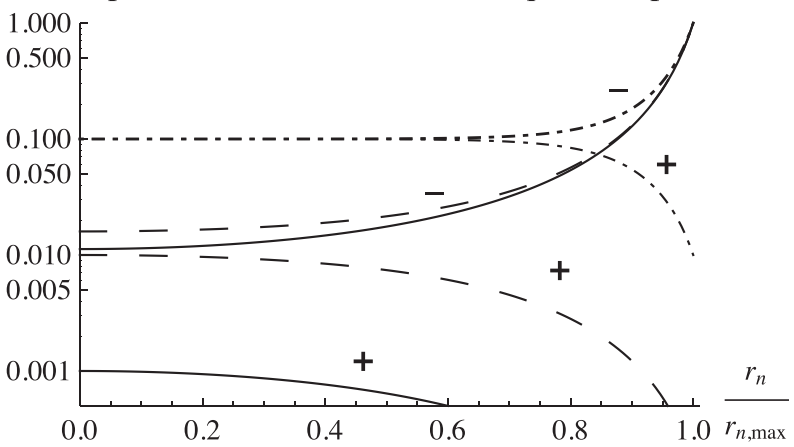

FIG. 7. Two plasma species with equal temperatures and charge states. The plots are for $T_{n}=1, r_{n, \max }=30$, and $N_{n}=0.1$ (dot-dashed), 0.01 (dashed), and 0.001 (solid). The \pm labels are defined in Fig. 5 .

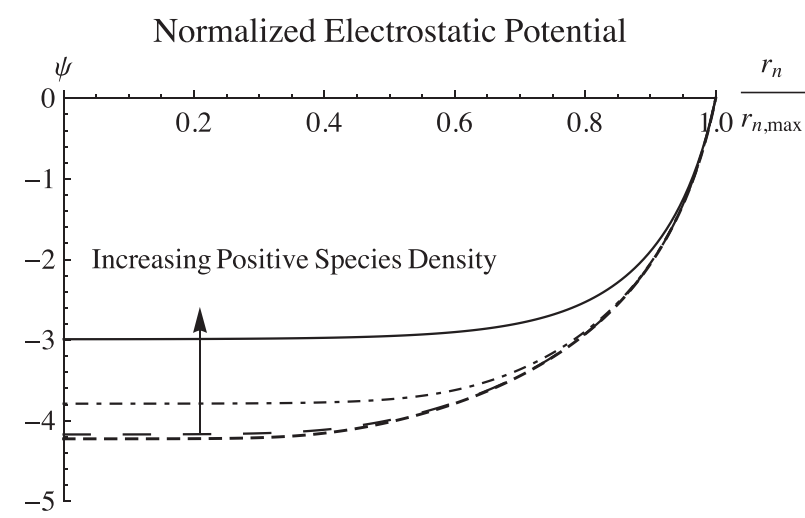

Two-Species Plasma Distribution: Equal Densities

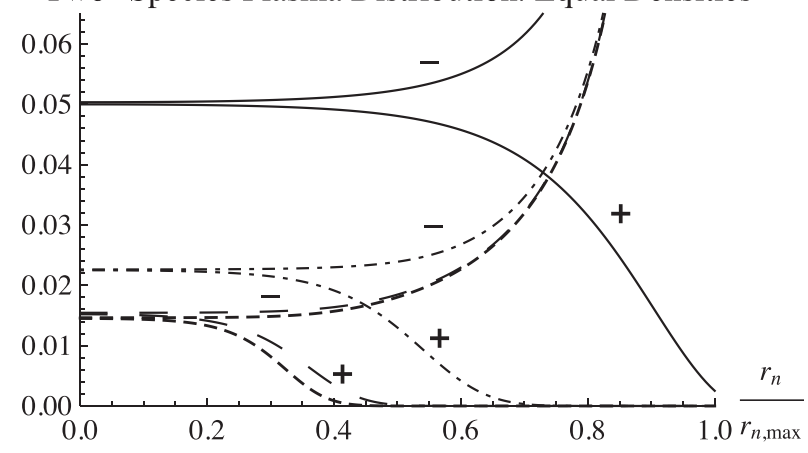

FIG. 8. Two plasma species with approximately equal charge densities at the center of the plasma system. The plots are for $r_{n, \max }=30$ and $\left(T_{n}, N_{n}\right)=(1,0.05)$ [solid], $(10,0.0225)$ [dot-dashed], $(25,0.0152)$ [long dash], and (40,0.0145)[short dash]. The normalized electron temperatures, $T_{n}$, were chosen and the normalized positive plasma charge densities, $N_{n}$, were then adjusted to the lowest value at which the two distributions have approximately the same value at the center of the system. The \pm labels are defined in Fig. 5.

\section{SPACE-CHARGE-BASED ELECTROSTATIC CONFINEMENT CONDITIONS}

Good confinement of the positive plasma species can be expected to occur when the electrostatic potential energy well that is self-consistently created by the plasma is much deeper than the temperature (in energy units) of the positive plasma species ${ }^{41}$

$$
T_{+} \ll Z e \Delta \phi_{0} .
$$

Here, $\Delta \phi_{0}=\phi\left(r_{\max }\right)-\phi(0)$ is the ordinary (unnormalized) electrostatic potential difference between the edge and the center of the configuration. It may also be possible to sustain the positive plasma species, even when Eq. (20) is not satisfied, provided that sufficient fueling and heating are used. In terms of a normalized quantity, the condition is written as

$$
\Delta \psi_{+0, \alpha}=\frac{Z e \Delta \phi_{0}}{T_{+}} \gg 1 .
$$

$\Delta \psi_{+0, \alpha}$ is the normalized electrostatic potential energy well depth that confines the positive plasma, and the value of the subscript $\alpha$ is used to indicate the geometry considered. $\Delta \psi_{+0, \alpha}$ is evaluated in Fig. 9. A value for $\Delta \psi_{+0, \alpha}$ is first obtained by employing the approximate expression 

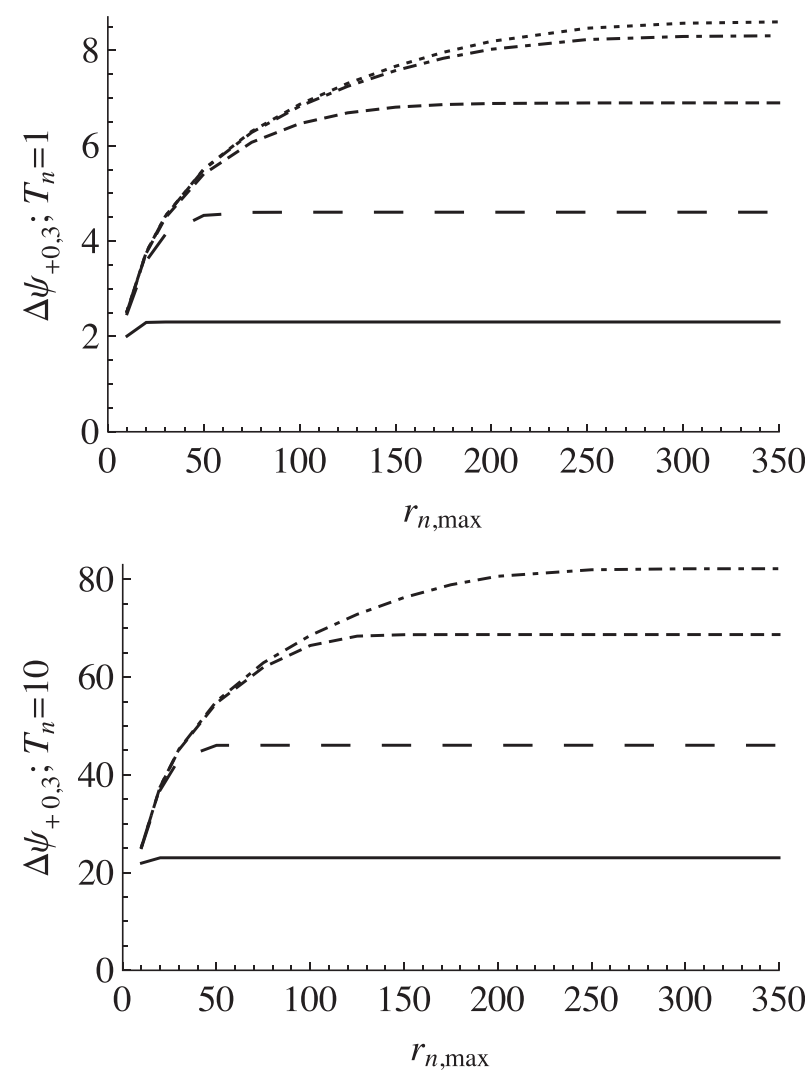

FIG. 9. Normalized electrostatic potential energy well depth for spacecharge-based electrostatic plasma confinement as a function of normalized system size. The dotted line in the top panel is for $N_{n}=0$ and $T_{n}=1$. Top (bottom) panel is for $T_{n}=1\left(T_{n}=10\right)$, and $N_{n}=0.1$ (solid), 0.01 (long dash), 0.001 (dash), and 0.0001 (dotted-dashed).

developed in Sec. II [Eq. (14)], which represents the limit $N_{n} \rightarrow 0$. The result is shown in Fig. 9 (top panel, dotted line). To explore the effect that $T_{n}$ and $N_{n}$ have on $\Delta \psi_{+0, \alpha}$, this quantity was evaluated as a function of system size for two different values of $T_{n}$ and varying $N_{n}$ values. It is found that the normalized electrostatic potential energy well depth tends to decrease as the normalized charge density of the positive plasma is increased, and to increase with normalized system size. However, for a sufficiently large normalized size, the normalized well depth saturates to a value nearly independent of the normalized size of the system. The normalized well depth is also found to increase with $T_{n}$.

Figure 10 shows $\Delta \psi_{0, \alpha}=\Delta \psi_{+0, \alpha} / T_{n}=e \Delta \phi_{0} / T_{-}$, the normalized electrostatic potential difference, evaluated in the saturated regime for several values of $T_{n}$ and $N_{n}$. An expression for the normalized electrostatic potential difference is readily derived by assuming that the plasma is neutral at the center. The expression is

$$
\frac{\Delta \psi_{+0, \alpha}}{T_{n}}=\Delta \psi_{0, \alpha}=-\ln \left(N_{n}\right)
$$

Inspection of Figs. 9 and 10 indicates that, for the spherical geometry, Eq. (22) is applicable for $r_{n, \max } \gtrsim 200,10^{-4} \lesssim N_{n}$ $<1$, and $1 \leq T_{n} \leq 10$. The close agreement between

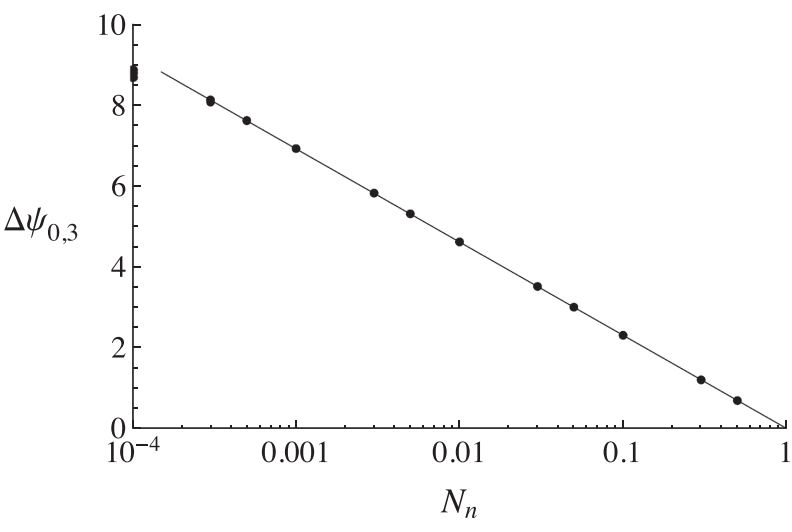

FIG. 10. Normalized electrostatic potential difference for increasing normalized charge density of the positive species. There are plot points for $r_{n, \max }=$ 300 and $T_{n}=1,4$, and 10 , for each value of $N_{n}$, but the plot points are indistinguishable. The solid line is Eq. (22).

Eq. (22) and the numerical values suggests that the saturated regime is an equilibrium in which a neutral region forms. It is conjectured that the electric potential well depth in the two-species plasma is given by the smaller of Eqs. (14) and (22). The predicted electric potential well depth can be used together with collision-based theory, such as that in Refs. 41 and 42 , to evaluate space-charge-based electrostatic plasma confinement timescales.

\section{CONCLUSION}

A self-consistent computation of the electrostatic potential generated by an edge-confined or surface-emitted non-neutral plasma that follows a Boltzmann density distribution with planar, cylindrical, or spherical symmetry has been carried out. The electrostatic potential profile, plasma density distribution, and the electrostatic potential well depth for different values of the normalized (to the edge Debye length) plasma size have been evaluated. Relatively deep electrostatic potential wells are predicted by the computation when the plasma size is much greater than the Debye length. An approximate expression has been fitted for the electrostatic potential well depth as a function of the normalized plasma size.

A self-consistent computation has been carried out for a positive plasma that is confined by the space charge of an edge-confined or surface-emitted electron plasma, with both species following Boltzmann density distributions. The results also apply to a negative plasma that is confined by the space charge of an edge-confined positive plasma such as a positron plasma. The results presented for the two-species system pertain to a spherically symmetric system, but are expected to be qualitatively applicable to planar and cylindrical geometries. The two-species system has been characterized by varying normalized parameters, which include (1) the ratio of the plasma radius to the Debye length at the plasma edge, (2) the ratio of the positive plasma charge density at the center of the system to that of the electron plasma at the edge, and (3) the ratio of the temperatures multiplied by the average charge state. The electrostatic potential profile and the charge density distribution have been computed, and the cases explored indicate the following: (1) Increasing 
the charge density of the positive plasma species decreases the depth of the electrostatic potential well. (2) Increasing the temperature or decreasing the average charge state of the positive plasma causes the positive plasma to occupy a larger volume. (3) An equilibrium is possible in which the two plasma species have equal temperatures and equal charge states. (4) Approximately, equal charge densities of the two plasma species at the center of the system occurs for a sufficiently high charge density of the positive plasma species, even when the temperatures and charge states are equal.

The electrostatic potential well depth has been evaluated for the two-species system. It has been found that, once the positive plasma is introduced, the electrostatic potential well depth reaches a saturation regime when the normalized plasma size is sufficiently large.

\section{ACKNOWLEDGMENTS}

The authors would like to thank J. R. Rocha for helpful suggestions. This material is based upon work supported by the Department of Energy under Grant No. DE-FG0206ER54883 and by the National Science Foundation under Grant No. PHY-1202428.

${ }^{1}$ J. U. Andersen, P. Hvelplund, S. B. Nielsen, S. Tomita, H. Wahlgreen, S. P. Møller, U. V. Pedersen, J. S. Forster, and T. J. D. Jørgensen, Rev. Sci. Instrum. 73, 1284 (2002).

${ }^{2}$ T. Tanabe, K. Chida, K. Noda, and I. Watanabe, Nucl. Instrum. Methods Phys. Res. A 482, 595 (2002).

${ }^{3}$ S. Jinno, T. Takao, K. Hanada, M. Goto, K. Okuno, H. Tanuma, T. Azuma, and H. Shiromaru, Nucl. Instrum. Methods Phys. Res. A 572, 568 (2007).

${ }^{4}$ D. Zajfman, O. Heber, L. Vejby-Christensen, I. Ben-Itzhak, M. Rappaport, R. Fishman, and M. Dahan, Phys. Rev. A 55, R1577 (1997).

${ }^{5}$ M. Dahan, R. Fishman, O. Heber, M. Rappaport, N. Altstein, D. Zajfman, and W. J. van der Zande, Rev. Sci. Instrum. 69, 76 (1998).

${ }^{6}$ H. T. Schmidt, H. Cederquist, J. Jensen, and A. Fardi, Nucl. Instrum. Methods Phys. Res. B 173, 523 (2001)

${ }^{7}$ T. Suzuki and Y. Yamauchi, Nucl. Instrum. Methods Phys. Res. A 562, 53 (2006).

${ }^{8}$ K. G. Bhushan, S. C. Gadkari, J. V. Yakhmi, and V. C. Sahni, Rev. Sci. Instrum. 78, 083302 (2007)

${ }^{9}$ H. Wollnik, A. Casares, D. Radford, and M. Yavor, Nucl. Instrum. Methods Phys. Res. A 519, 373 (2004).

${ }^{10}$ Y. Ishida, M. Wada, and H. Wollnik, Nucl. Instrum. Methods Phys. Res. B 241, 983 (2005).

${ }^{11}$ K. H. Kingdon, Phys. Rev. 21, 408 (1923).
${ }^{12}$ D. P. Moehs, D. A. Church, and R. A. Phaneuf, Rev. Sci. Instrum. 69, 1991 (1998).

${ }^{13}$ S. Robertson and D. Alexander, Phys. Plasmas 2, 3 (1995).

${ }^{14}$ C. A. Ordonez, Phys. Plasmas 15, 072508 (2008).

${ }^{15}$ A. M. Clogston and H. Heffner, J. Appl. Phys. 25, 436 (1954).

${ }^{16}$ P. K. Tien, J. Appl. Phys. 25, 1281 (1954).

${ }^{17}$ C. A. Ordonez, J. Appl. Phys. 104, 054903 (2008).

${ }^{18}$ C. A. Ordonez, IEEE Trans. Plasma Sci. 38, 388 (2010).

${ }^{19}$ R. E. Marrs, M. A. Levine, D. A. Knapp, and J. R. Henderson, Phys. Rev. Lett. 60, 1715 (1988).

${ }^{20}$ E. D. Donets, in The Physics and Technology of Ion Sources, edited by I. G. Brown (Wiley, New York, 1989), p. 245.

${ }^{21}$ C. A. Ordonez, J. Appl. Phys. 94, 3732 (2003).

${ }^{22}$ C. A. Ordonez, Phys. Rev. E 67, 046401 (2003).

${ }^{23}$ D. D. Dolliver and C. A. Ordonez, Phys. Rev. E 62, 5855 (2000).

${ }^{24}$ Y. Gu and G. H. Miley, IEEE Trans. Plasma Sci. 28, 331 (2000).

${ }^{25}$ D. C. Barnes, M. M. Schauer, K. R. Umstadter, L. Chacon, and G. Miley, Phys. Plasmas 7, 1693 (2000).

${ }^{26}$ A. Mohri and Y. Yamazaki, Europhys. Lett. 63, 207 (2003).

${ }^{27}$ A. Mohri, T. Yuyama, Y. Kiwamoto, Y. Yamazawa, and T. Michishita, Jpn. J. Appl. Phys., Part 2 37, L1553 (1998).

${ }^{28}$ J. L. Pacheco, C. A. Ordonez, and D. L. Weathers, IEEE Trans. Plasma Sci. 39, 2424 (2011).

${ }^{29}$ C. A. Ordonez and R. M. Hedlof, AIP Adv. 2, 012176 (2012).

${ }^{30}$ L. V. Jørgensen et al., "The AEgIS antihydrogen gravity experiment," Hyperfine Interact. (in press).

${ }^{31}$ G. B. Andresen, M. D. Ashkezari, M. Baquero-Ruiz, W. Bertsche, P. D. Bowe, E. Butler, C. L. Cesar, S. Chapman, M. Charlton, A. Deller, S. Eriksson, J. Fajans, T. Friesen, M. C. Fujiwara, D. R. Gill, A. Gutierrez, J. S. Hangst, W. N. Hardy, M. E. Hayden, A. J. Humphries, R. Hydomako, M. J. Jenkins, S. Jonsell, L. V. Jørgensen, L. Kurchaninov, N. Madsen, S. Menary, P. Nolan, K. Olchanski, A. Olin, A. Povilus, P. Pusa, F. Robicheaux, E. Sarid, S. Seif el Nasr, D. M. Silveira, C. So, J. W. Storey, R. I. Thompson, D. P. van der Werf, J. S. Wurtele, and Y. Yamazaki, Nature (London) 468, 673 (2010).

${ }^{32}$ G. Gabrielse, R. Kalra, W. S. Kolthammer, R. McConnell, P. Richerme, D. Grzonka, W. Oelert, T. Sefzick, M. Zielinski, D. W. Fitzakerley, M. C. George, E. A. Hessels, C. H. Storry, M. Weel, A. Mullers, and J. Walz, Phys. Rev. Lett. 108, 113002 (2012).

${ }^{33}$ C. A. Ordonez and D. L. Weathers, Phys. Plasmas 15, 083504 (2008).

${ }^{34}$ C. A. Ordonez, Phys. Rev. E 76, 017402 (2007).

${ }^{35}$ C. A. Ordonez, D. D. Dolliver, Y. Chang, and J. R. Correa, Phys. Plasmas 9, 3289 (2002).

${ }^{36}$ M. E. Glinsky and T. M. O’Neil, Phys. Fluids B 3, 1279 (1991).

${ }^{37}$ R. L. Spencer, S. N. Rasband, and R. R. Vanfleet, Phys. Fluids B 5, 4267 (1993).

${ }^{38}$ W. H. Press, S. A. Teukolsky, W. T. Vetterling, and B. P. Flannery, $N u$ merical Recipes: The Art of Scientific Computing (Cambridge University Press, Cambridge, 2007), p. 1062.

${ }^{39}$ A. J. Peurrung and J. Fajans, Phys. Fluids B 2, 693 (1990).

${ }^{40}$ S. A. Prasad and T. M. O'Neil, Phys. Fluids 22, 278 (1979).

${ }^{41}$ Y. Chang, J. R. Correa, and C. A. Ordonez, Phys. Plasmas 11, 3360 (2004).

${ }^{42}$ J. R. Correa, Y. Chang, and C. A. Ordonez, Phys. Plasmas 12, 084505 (2005). 